\title{
Pessoas com Deficiência no Trabalho: Análise da Percepção de Pessoas sem Deficiência
}

\section{People with Disabilities at Work: Analysis of the Perception of People without Disabilities}

\author{
Renata Réab; Marilsa de Sá Rodrigues*b \\ ${ }^{a}$ Universidade de Taubaté, SP, Brasil \\ ${ }^{\mathrm{b}}$ Anhanguera Educacional, SP, Brasil. \\ 'Universidade de Taubaté, Departamento de Psicologia. SP, Brasil. \\ *E-mail: marilsasarodrigues@outlook.com
}

\begin{abstract}
Resumo
O presente trabalho tem como objetivo identificar a concepção da percepção de pessoas sem deficiência sobre pessoas com deficiência (PcD), e sua interação no contexto de trabalho. A pesquisa de natureza descritiva teve como instrumentos o Inventário de Concepções de Deficiência em Situações de Trabalho (ICD-ST) e entrevista semiestruturada, em pessoas sem deficiência. A amostra foi composta por 37 alunos, dos cursos presenciais de Pós-Graduação da UNITAU, que trabalham com PcD. Os resultados obtidos da relação desses alunos com os PcDs apontam que o constructo "normalidade" foi o mais pontuado, demonstrando dessa maneira que há uma tendência de exclusão social das PcD; porém é importante para as empresas contratarem PcD, pois obtêm benefícios para sua imagem. Nas entrevistas, apesar da interação demonstrar boa qualidade, conclui-se na integração dos dados coletados que a visão das pessoas sem deficiência, que prevalece sobre as PcD, é a de que deveriam se manter segregadas, não tanto pelas suas limitações, mas principalmente, conforme relatado, que as organizações procurando cumprir as Cotas determinadas por lei, se esquecem da necessidade de adaptações e sensibilização para melhor recebê-las nas organizações.
\end{abstract}

Palavras-chave: Pessoa com Deficiência. Inclusão. Organização.

\begin{abstract}
The present work has as objective to identify the conception of the perception of people without disability about people with disability (PcD), and their interaction at work context. The research of descriptive nature had as instruments the Inventory of Conceptions of Disability at Work Situations (ICD-ST) and half-structured interview, with people without disability. The sample was composed of 37 students, from the classroom courses of Post-Graduation of UNITAU, who work with PcD. The results obtained from the relationship between these students and PcDs, point out that the construct "normality" was the most pointed one, showing thus, that there is a tendency of social exclusion of PcD; however, it is important to the companies, to hire PcD, because they obtain benefits to their images. During the interviews, despite the interaction showed good quality, it could be concluded that on the integration of the collected data the vision of people without disability, which prevails over the $P c D$, is that they should remain segregated, not so much by their limitations, but mainly, as related, because the companies trying to accomplish the Quotas defined by law, forget the need of adaptations and awareness to better receive them at the companies.
\end{abstract}

Keywords: People with Disabilities. Inclusion. Organization.

\section{Introdução}

As organizações buscam profissionais que tenham algo a lhes oferecer no sentido de esforço e trabalho, para o cumprimento de metas individuais e coletivas. A gestão desses profissionais transforma o trabalho em resultado. Sendo assim, é necessário o preparo dos profissionais para lidar com diferenças individuais na equipe. Destas diferenças se destaca aqui a Pessoa Com Deficiência (PcD), não no sentido de ser diferente fisicamente, por exemplo, mas por caracterizar a necessidade de um preparo no trato com as diferenças, no que se vê como atitudes de inclusão e das práticas de gestão, que facilitem a relação de trabalho entre as pessoas.

O presente trabalho tem como objetivo identificar a concepção da percepção de pessoas sem deficiência sobre pessoas com deficiência ( $\mathrm{PcD}$ ), e sua interação no contexto de trabalho. A pesquisa de natureza descritiva teve como instrumentos o Inventário de Concepções de Deficiência em Situações de Trabalho (ICD-ST) e entrevista semiestruturada, com pessoas sem deficiência.

\section{Material e Métodos}

Os métodos de pesquisa foram Estudo Descritivo, de Abordagem Quantitativa e Qualitativa, por Levantamento. A população e amostra foram alunos de todas as 50 (cinquenta) turmas dos cursos presenciais de Pós-Graduação da Universidade de Taubaté (UNITAU), com um total de 825 (oitocentos e vinte e cinco) alunos. Entre esses, 63 (sessenta e três) se identificaram como que trabalham com pessoas com deficiência nas organizações e 37 (trinta e sete) aceitaram participar da pesquisa.

Os intrumentos foram: o Inventário de Concepções de Deficiência em Situações de Trabalho (ICD-ST), que inicia com as instruções de preenchimento, composto por 19 (dezenove) afirmativas que deverão ser respondidas entre 05 (cinco) escalas - Concordo Totalmente até Discordo Totalmente; o Inventário de Assertividade que também inicia com as instruções de preenchimento, composto por 35 (trinta 
e cinco) questões, sendo respondidas entre Sim, Às vezes e Não; e a entrevista semiestruturada baseada no que diz respeito aos aspectos da situação da pessoa com deficiência na organização dos entrevistados.

Para o cumprimento das etapas desta pesquisa, a coleta de dados ocorreu, inicialmente, pela autorização da diretoria dos cursos de Pós-Graduação para pesquisa com alunos, e foi apresentado junto ao Comitê de Ética em Pesquisa da UNITAU para aprovação, e então ser formalizado o encontro com apresentação do questionamento junto aos alunos que trabalham com PcD, com explicação do objetivo e procedimento da pesquisa para adesão, em seguida à orientação foi realizada a aplicação dos inventários e entrevista, mediante permissão, e entrevista individual, realizada ao final do preenchimento dos inventários.

A análise de dados do Inventário e de conteúdo da entrevista ocorreram com base nos crivos determinado pelos respectivos autores. Quanto à construção do relatório dos dados coletados ao longo do período de agosto a setembro de 2013, esse teve a utilização do software MS-Excel e SPSS.

\section{Resultados e Discussão}

\subsection{A realidade da pessoa com deficiência}

Segundo o censo realizado em 2010, pelo IBGE - Instituto Brasileiro de Geografia e Estatística - são mais de 190 milhões de pessoas a população brasileira, sendo mais de 45 milhões deles com algum tipo de deficiência, o que corresponde a $24 \%$ da população brasileira. No Estado de São Paulo são mais de 35 milhões de pessoas, e 09 milhões de $\mathrm{PcD}$, correspondendo à $21 \%$.

Para descrever Deficiência, o Decreto $\mathrm{n}^{\circ} 3.298$, de 20 de dezembro de 1999, considera: Art. $3^{\circ}$ Para os efeitos deste Decreto considera-se: I - deficiência - toda perda ou anormalidade de uma estrutura ou função psicológica, fisiológica ou anatômica que gere incapacidade para o desempenho de atividade, dentro do padrão considerado normal para o ser humano.

A convivência com as diferenças é condição da vida em sociedade, e seu alicerce deve ser o bom senso e respeito, que consideram as particularidades de cada pessoa, independente de suas limitações. Segundo Barbosa et al. (2009), a Pessoa com Deficiência é vitimada pelo preconceito e pela intolerância diante da diversidade, de situações resultantes da ignorância e da falta de informação. Quanto a este aspecto, a nomenclatura ou a maneira como são designadas as pessoas com deficiência tem total importância na diversidade e inclusão. Como se denomina, conceitua e qual terminologia se utiliza para designar a PcD têm se alterado ao longo dos anos. Por várias vezes, ouvem-se expressões pejorativas e que estão relacionadas aos conceitos de cultura e época. Conforme ressalta Rebelo (2008, p.35): “Até meados do século passado, as pessoas com deficiência eram consideradas incapazes e entregues à caridade de familiares ou instituições religiosas", portanto, segregadas da sociedade.

O conceito tem se misturado a conteúdos de terminologia diversos, como: aleijado, defeituoso, incapacitado, inválido, pessoa portadora de deficiência, pessoas com necessidades especiais, e por fim pessoas com deficiência, que segundo descreve Sassaki (2003) eram termos utilizados até a década de 1980, e com a influência do Ano Internacional das Pessoas Deficientes - realizado pela Organização das Nações Unidas (ONU), a expressão pessoa deficiente passou a ser falada e escrita pela primeira vez. Já a expressão pessoa portadora de deficiência foi introduzida aos poucos. Fávero (2004) questiona o termo "portador" enfatizando que seria melhor incluir o "com", pessoas "com" deficiência, como também o termo deficiência não deve ser substituído por "necessidade especial", já que a palavra deficiência não deve gerar aspecto negativo, até porque se fala de seres humanos.

No Brasil, entre 1986 e 1996, o termo "portador de deficiência" se tornou popular. Em meados da década de 1990 se passou a usar o termo "pessoas com deficiência", permanecendo até hoje. Este termo pondera "que elas não portam deficiência; que a deficiência que elas têm não é como coisas que às vezes portamos e às vezes não portamos. $\mathrm{O}$ termo preferido passou a ser pessoa com deficiência" (Sassaki, 2003, p. 160).

Segundo Schwarz e Haber (2009, p.84): "Essa conceituação alinha-se aos preceitos de sociedade inclusiva, na qual é a sociedade que não se ajustou, ainda, para favorecer a liberdade, o exercício dos direitos e a autonomia de toda a diversidade humana." Para Aranha (2003), a sociedade em relação à pessoa com deficiência pode se considerar inclusiva, quando a diversidade que a constitui é reconhecida, e quando identifica as necessidades desta população, oferecendo para suas necessidades serviços, que promovam ajustes para viabilizar o acesso, utilização e permanência nos espaços públicos.

\subsection{Perfil da população e amostra}

Para os alunos dos 825 cursos em andamento no momento da coleta de dados, nota-se que entre estes 703 alunos, do total de turmas abordadas, somente 63 se identificaram como que trabalham com $\mathrm{PcD}$, totalizando $9 \%$. Em relação aos que aceitaram responder a pesquisa - 37, o percentual é ainda menor, totalizando $5 \%$. Entre os que se identificaram com relação aos respondentes a diferença chega a 59\%.

O baixo índice de identificação e participação na pesquisa levanta a questão, se as organizações nas quais todos os alunos atuam cumprem a Lei de Cotas, Decreto n ${ }^{\text {o } 3.298, ~ d e ~}$ 20 de Dezembro de 1999, que regulamenta a Lei $\mathrm{n}^{\circ} 7.853$, de 24 de outubro de 1989, que obriga as empresas com 100 ou mais empregados, a preencher o quadro de funcionários com dois a cinco por cento de seus cargos com PcD; ou, se os tipos de deficiência das PcD contratadas são imperceptíveis, apesar das definições da mesma Lei, Deficiência, o Decreto ${ }^{\circ}$ 
3.298, de 20 de dezembro de 1999, considera: Art. $3^{\circ}$ Para os efeitos deste Decreto considera-se: I - deficiência - toda perda ou anormalidade de uma estrutura ou função psicológica, fisiológica ou anatômica que gere incapacidade para o desempenho de atividade, dentro do padrão considerado normal para o ser humano.

Segundo o Censo do IBGE de 2010, o número de pessoas ocupadas com carteira assinada no Brasil chegava a mais de 39 milhões, totalizando $45 \%$, e pessoas ocupadas sem carteira perfaziam mais de 47 milhões, que corresponde a 55\%. Com referência às pessoas com deficiência, os dados do Censo apresentam mais de 3,5 milhões de PcD ocupadas com carteira assinada no Brasil e mais de 16 milhões de PcD ocupadas sem carteira, sendo respectivo de $18 \%$ e $82 \%$.

A exclusão social que a $\mathrm{PcD}$ vive está relacionada com a falta de conhecimento e pré-concepções das suas condições de vida e necessidades. Nestes aspectos é apresentada a inclusão social através do trabalho e acessibilidade. Para Sassaki (1997), a inclusão social é conceituada como um processo de adaptação pelo qual a sociedade passa para poder incluir pessoas com deficiência, em seus sistemas sociais, e estas se preparam para assumir papéis na sociedade. Para Marques (2001), ser uma empresa inclusiva significa acreditar na diversidade humana e fazer o possível para que as PcD se adaptem ao ambiente de trabalho.

As empresas, ao se obrigarem a contratar, se dão conta não só da falta de conhecimento das necessidades das $\mathrm{PcD}$, como também da dificuldade de recebê-las em seus ambientes (local de trabalho, áreas comuns - banheiro, refeitório). Nestes aspectos, aparece a necessidade de acessibilidade, enfatizada no Decreto n 5.296 de 02 de Dezembro de 2004: condição para utilização, com segurança e autonomia, total ou assistida, dos espaços, mobiliários e equipamentos urbanos, das edificações, dos serviços de transporte e dos dispositivos, sistemas e meios de comunicação e informação, por pessoa portadora de deficiência ou com mobilidade reduzida.

As adequações sobre acessibilidade pontuadas na lei facilitam o acesso das PcD aos locais de trabalho, como também o usufruto do que o emprego, com salário e benefícios lhe proporciona. Segundo Schwarz e Haber (2009), a acessibilidade: são todos recursos que possibilitam o acesso e o uso de modo seguro e autônomo, aos espaços, mobiliários e equipamentos urbanos, às edificações, aos meios de transporte e aos sistemas e meios de comunicação. A igualdade de oportunidades para a $\mathrm{PcD}$, em todos os âmbitos, fortalece o conceito de acessibilidade e as ações de inclusão social.

A Superintendência Regional do Trabalho e Emprego de São Paulo (SRTE-SP) realizou um mirante da inclusão das pessoas com deficiência no mercado de trabalho formal do Estado de São Paulo, conforme Quados 1 e 2. Nestas, constam o número e um comparativo de contratos de pessoas com deficiência, conforme a lei de cotas, na RAIS (Relação Anual de Informações Sociais) em 2009 e 2010.
Quadro 1- $\mathrm{N}^{\mathrm{o}}$ de contratos de pessoas com deficiência - RAIS 2009.

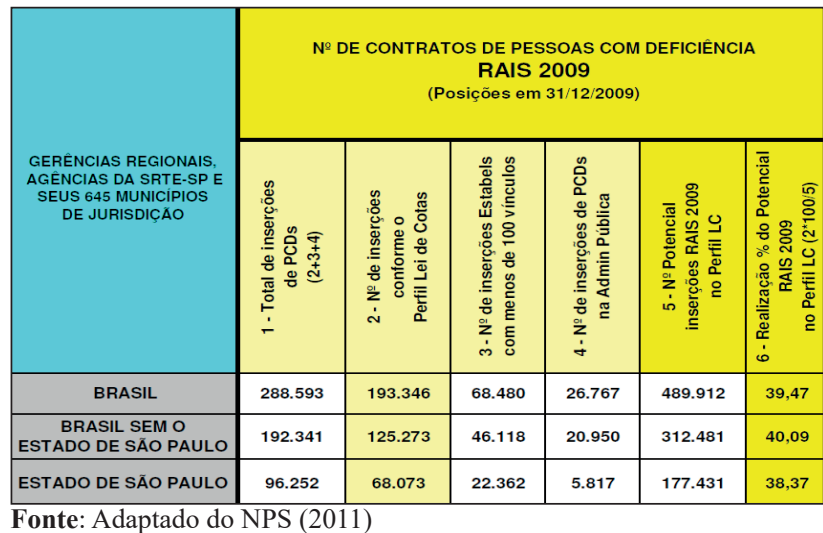

Quadro 2 - $\mathrm{N}^{\mathrm{o}}$ de contratos de pessoas com deficiência - RAIS 2010 .

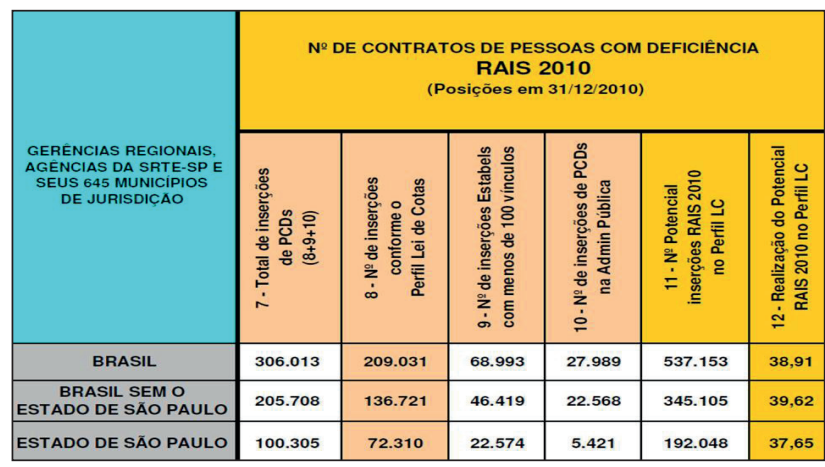

Fonte: Adaptado do NPS (2011)

Observando estes dados se verifica um aumento de inserções no Brasil, de PcD no trabalho com e sem menção ao Estado de São Paulo, tanto em empresas com mais de 100 funcionários, que têm a cobrança da lei de Cotas, quanto em empresas sem esta cobrança, com menos de 100 funcionários. Mesmo que pequeno, esse aumento demonstra a ação das empresas em relação à inclusão social pelo trabalho, e ainda mais importante, a contratação por empresas que não têm a obrigatoriedade pela Lei de Cotas. No entanto, ainda, segundo a RAIS-2010, apenas 14\% das empresas cumprem a Lei de Cotas.

Segundo Rebelo (2008), a integração da pessoa com deficiência na sociedade ocorre pela inclusão pelo trabalho, mas na maioria das vezes lhe falta preparo pessoal e técnico, o que o faz buscar por seus próprios esforços se integrar. A falta de suporte educacional e de saúde, por parte das políticas públicas, remete a $\mathrm{PcD}$ ao despreparo técnico e pessoal, para concorrer a vagas de emprego que lhe proporcionem carreiras promissoras.

Em relação à educação no Ensino Superior, Pereira (2011) ressalta que a maior conscientização promovida por organizações não governamentais e movimentos sociais, e novas políticas de inclusão, têm aumentado o número de pessoas com deficiência no Ensino Superior. Fator que é reforçado com a preocupação ao cumprimento das leis e com a conscientização das $\mathrm{PcD}$ e de suas famílias do quão 
produtivos podem ser.

A pessoa com deficiência é um indivíduo que é visto com as perspectivas reduzidas de obter um emprego adequado, de assumi-lo, mantê-lo e progredir, em função da deficiência física, auditiva, visual, mental ou múltipla devidamente reconhecida, e que são agravadas pelas constantes dificuldades de inclusão no mundo do trabalho.

Relativo às 42 turmas abordadas neste estudo, 28 cursos trabalham ou trabalharam com PcD, e os que participaram da pesquisa. Os cursos que mais se identificaram foram os relacionados às áreas de Engenharia. Em relação ao perfil dos participantes se levantou como gênero masculino 21 participantes e 16 feminino. No tipo de organização que trabalham prevaleceu a Indústria, com 13, seguido pelo Ensino com 11, Serviços com 07, Público com 05 e Saúde com um.

\subsection{Dados de Concepções de deficiência em situações de trabalho}

As concepções de deficiência são modos de pensamento construídos ao longo da história, são modelos ou tipos ideais, são formas de interpretação da deficiência identificadas por meio de seus modelos explicativos sobre a deficiência e de seu impacto na inserção das pessoas com deficiência (CARVALHO-FREITAS, 2007, p.2).

A autora realizou uma pesquisa, e desenvolveu quatro matrizes, que detalham as concepções de deficiência, construídas com base em estudos de períodos históricos, que mencionaram as PcD. Após análise se verificou predomínio de determinados padrões de comportamento em relação à $\mathrm{PcD}$. Com base nos dados coletados na pesquisa foram descritas 04 matrizes de concepções de deficiência (Quadro 3):

Quadro 3 - Matrizes de Concepções de deficiência

\begin{tabular}{|c|c|}
\hline Matrizes & Concepções de Deficiência \\
\hline $\begin{array}{l}\text { A deficiência } \\
\text { vista como } \\
\text { fenômeno }\end{array}$ & $\begin{array}{l}\text { - A característica principal das pessoas que a possuem é atribuir uma origem metafísica à deficiência e considerá- } \\
\text { la uma manifestação de desejos ou castigos divinos. } \\
\text { - A pessoa com deficiência, nesta matriz, é considerada como alguém que precisa ser mantida e cuidada, } \\
\text { contribuindo para o surgimento de sentimentos de caridade e compaixão em relação a elas. }\end{array}$ \\
\hline $\begin{array}{l}\text { A normalidade } \\
\text { como } \\
\text { matriz de } \\
\text { interpretação }\end{array}$ & $\begin{array}{l}\text { - As pessoas que compartilham desta concepção têm na norma seu padrão de avaliação, sendo a deficiência } \\
\text { considerada um "desvio" ou "doença" que necessita de cuidados especiais dos profissionais da saúde. } \\
\text { - Para as pessoas que possuem esta visão da deficiência, a possibilidade de inserção das pessoas com deficiência } \\
\text { ocorre mediante a reabilitação e a adequação delas ao sistema social. } \\
\text { - Geralmente, as pessoas que compartilham desta concepção consideram que as pessoas com deficiência assumem } \\
\text { atitudes inadequadas no trabalho, provocando situações embaraçosas, são mais propensas a acidentes, têm } \\
\text { problemas de relacionamento e devem ser alocadas em setores específicos da empresa. } \\
\text { - Além disso, consideram as instituições especializadas mais adequadas ao trabalho das pessoas com deficiência. }\end{array}$ \\
\hline $\begin{array}{l}\text { A inclusão } \\
\text { como } \\
\text { matriz de } \\
\text { interpretação }\end{array}$ & $\begin{array}{l}\text { - Esta concepção é compartilhada pelas pessoas que deslocam sua percepção da deficiência de um problema } \\
\text { individual para um problema social. } \\
\text { - O pressuposto compartilhado pelas pessoas que possuem esta visão da deficiência é de que a sociedade tem } \\
\text { que se adaptar para incluir a todos e que a inclusão das pessoas com deficiência deve ser feita a partir de suas } \\
\text { potencialidades. }\end{array}$ \\
\hline $\begin{array}{l}\text { A matriz de } \\
\text { interpretação } \\
\text { técnica da } \\
\text { deficiência }\end{array}$ & $\begin{array}{l}\text { Essa se materializa através da concepção das pessoas que veem a diversidade como um recurso a ser gerido nas } \\
\text { organizações, e que consideram as práticas sociais e organizacionais como veículos para a participação e inclusão } \\
\text { das pessoas com deficiência. Essa matriz se traduz em quatro fatores, que avaliam as possibilidades de trabalho das } \\
\text { pessoas com deficiência em relação à: } \\
\text { - Percepção de desempenho: como característica geral, este fator focaliza a percepção das pessoas em relação } \\
\text { ao desempenho, produtividade e qualidade de trabalho das pessoas com deficiência e suas implicaçães para a } \\
\text { competitividade da empresa. } \\
\text { - Percepção do vínculo: como característica geral, este fator focaliza a percepção das pessoas em relação ao } \\
\text { comprometimento e estabilidade no emprego das pessoas com deficiência. } \\
\text { - Percepção dos benefícios da contratação: este fator focaliza a percepção do impacto da contratação de pessoas } \\
\text { com deficiência para a imagem da empresa junto a funcionários e clientes e também para o clima da organização. } \\
\text { - Percepção sobre a necessidade de treinamento: este fator indica a percepção quanto à necessidade de treinamento } \\
\text { das chefias e funcionários para a inserção de pessoas com deficiência na empresa. }\end{array}$ \\
\hline
\end{tabular}
Fonte: Adaptado de Carvalho-Freitas (2007)

Para melhor visualização do construto das concepções de deficiência em situações de trabalho, Carvalho-Freitas e Marques (2010) apresentam o modelo em forma de Figura 1 pressupondo a correlação entre as dimensões.

Carvalho-Freitas e Marques (2010) ressaltam que a utilização desse construto originou o ICD-ST - Inventário de Concepções de Deficiência em Situações de Trabalho, criado para identificação da forma como a gestão de pessoas percebia as deficiências e a possibilidade de trabalho dessas pessoas nas organizações. Segundo pesquisa, Carvalho-Freitas e
Marques (2010) tiveram como objetivo a identificação e o teste empírico do construto, e foi constatado que entre as concepções acima apresentadas, o fator de Percepção sobre a necessidade de treinamento "não apresenta relação direta com o construto de concepções de deficiência" (p.122). Portanto, dos sete fatores apresentados para as concepções de deficiência em situações de trabalho, seis foram identificados como dimensões relevantes ao Inventário de concepções de deficiência em situações de trabalho (ICD-ST). São essas: dimensão baseada em pressupostos da Normalidade, 
benefícios da contratação, dimensão espiritual, vínculo, dimensão baseada em pressupostos da Inclusão e percepções do desempenho.

Figura 1 - Modelo de Concepções de Deficiência em Situações de Trabalho

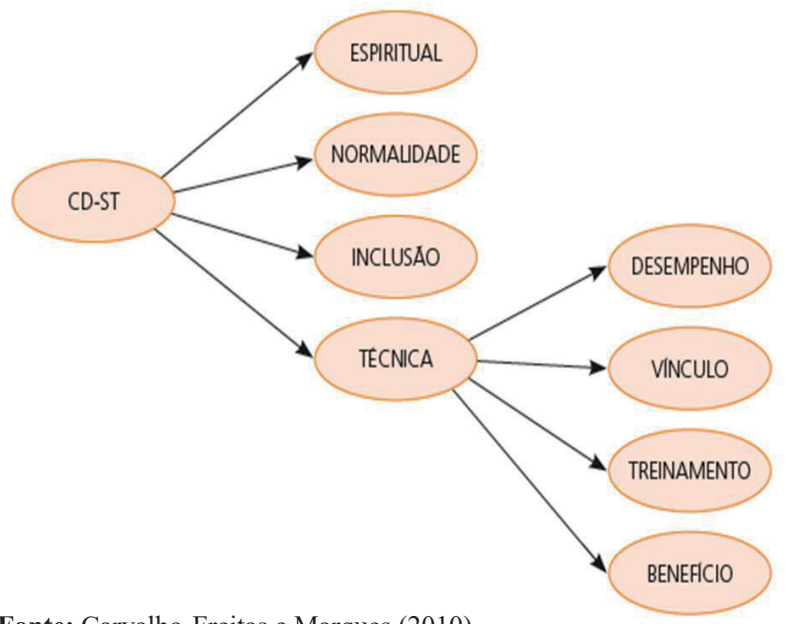

Fonte: Carvalho-Freitas e Marques (2010)

Carvalho-Freitas e Marques (2010) ressaltam ainda que, qualquer descrição da forma de ver a deficiência, mesmo a mais completa possível, nunca contemplará a complexidade envolvida na temática, embora seja aplicável a muitas pessoas.

Segundo dados da presente pesquisa, considerando os três maiores índices de pontuação, aparecem com maior predominância, a Dimensão baseada em pressupostos da Normalidade, Benefícios da Contratação, e Dimensão baseada em pressupostos da Inclusão. Sendo que, entre os 37 participantes, 23 deles a concepção de Dimensão baseada em pressupostos da Normalidade apareceu como predominante, totalizando $62 \%$ do total de participantes. Em 13 deles, ficou a Dimensão de Benefícios da Contratação, totalizando 35\%. Em apenas 01 aparece como predominante a Dimensão baseada em pressupostos da Inclusão, totalizando 3\%. Entre estas 03 dimensões há uma alternância entre primeiro, segundo e terceiro maiores índices. As demais concepções aparecem com pouca relevância, já que ficaram entre o quarto e sexto maiores índices.

O maior índice de Dimensão baseada em pressupostos da Normalidade demonstra a tendência das pessoas nas organizações de observarem as PcD, segundo CarvalhoFreitas (2007), como um desvio da normalidade ou doença, o que contribui para a segregação das pessoas com deficiência no trabalho. Portanto, a análise que se faz é de que as pessoas que compartilham da concepção de pressuposto da Normalidade acreditam que as $\mathrm{PcD}$ têm comportamentos inadequados no trabalho, o que provoca situações de embaraço, sendo também predispostas a acidentes e problemas de relacionamento, devendo então estar alocadas em setores diferenciados na organização, e acreditam, também, que seria mais adequado, se as PcD estivessem em instituições especializadas.

Levantando os resultados dos tres últimos índices de pontuação, aparecem: Vínculo, Dimensão espiritual e Percepção do desempenho como predominantes entre o quarto e o sexto índices. Sendo que, dos 37 participantes, 11 deles a concepção de Vínculo apareceu como predominante, totalizando 30\% do total de participantes. Em 10 deles ficou a Dimensão de Percepção do desempenho, totalizando 27\%. Em apenas 09 aparece como predominante a Dimensão espiritual, totalizando $24 \%$, destas 03 dimensões uma alternância entre primeiro, segundo e terceiro maiores índices. As demais concepções aparecem com pouca relevância, já que ficaram entre o primeiro e terceiro índices. Diante dos dados, entre o quarto e sexto índices, aparece pouca diferença entre seu percentual de predominância.

\subsection{Dados da entrevista semiestruturada}

Entre os aspectos que compõem as relações de trabalho nas organizações, a liderança tem papel fundamental para atingir as metas organizacionais através do apoio das equipes, dos processos estratégicos de gestão do trabalho, e das relações entre as pessoas. Segundo Miranda (2005), a liderança acredita que cria alternativas de contribuição para o desenvolvimento das pessoas, assim facilitando o atingimento das metas.

Conforme Spector (2010), condições físicas do trabalho trazem efeitos físicos nas pessoas. Estes aspectos de saúde nas organizações aparecem quando se fala em qualidade de vida no trabalho e ações de prevenção.

Com a captação do capital humano atrelado aos objetivos da organização se torna necessário a criação de meios que os mantenham em constante satisfação e desenvolvimento. Entre os métodos de Gestão de Pessoas está o Treinamento \& Desenvolvimento apresentado por Gil (2009) como um processo educacional para ampliar as capacidades das pessoas, focado nos conhecimentos, nas habilidades e nas atitudes dos funcionários, os cargos a serem ocupados, e aspectos das relações interpessoais, como trabalho em equipe, comunicação, administração de conflito, negociação. Assim, a possibilidade de adequação à realidade das organizações em relação às pessoas para atuar com as demandas técnicas e interpessoais de mercado está incorporada, legalmente, ao processo de contratação de pessoas com deficiência.

Diante destes aspectos, as entrevistas na pesquisa levantaram os seguintes dados:

$\mathrm{Na}$ pergunta 1, quanto ao tipo de deficiência, foram levantadas 04 categorias: Física $-81 \%$, Auditiva - 43\%, Intelectual $-24 \%$ e Visual - 13\%. A diferença entre a deficiência predominante - física, para as demais, pode caracterizar a falta de acessibilidade dessas aos ambientes, que possibilitem a busca pelo trabalho; como as escolas e a cursos profissionalizantes. Segundo a Declaração de Salamanca, elaborada na Conferência Mundial sobre necessidades educativas especiais, realizada em 1994, em Salamanca na Espanha, foram criadas diretrizes para os sistemas educacionais, e que fundamenta a escola inclusiva, em que o acesso à escola seja compartilhado a todas as crianças, 
aprendendo juntas com suas dificuldades e diferenças.

Na segunda pergunta, sobre o tipo de relação, levantou-se que as $\mathrm{PcD}$ trabalham, no Mesmo departamento - 43\% e/ou em Outro departamento - $57 \%$.

$\mathrm{Na}$ pergunta 03, quanto à função e departamento, foram levantados 02 setores: Administrativo - 78\% e/ou Produção $27 \%$, sendo que os participantes atuam diretamente nas áreas administrativas. As funções são as mais diversas, de acordo com os diferentes tipos de organização: Docente, Auxiliar/ Assistente Administrativo, Analistas, Supervisão e Diretoria.

$\mathrm{Na}$ faixa etária (pergunta 04) foram levantados 03 grupos: 14 a 30 anos, 31 a 50 anos e acima de 51, podendo apresentar, de acordo com a caracterização histórica do conceito e aceitação da deficiência, o início da movimentação para inclusão, em meados do início do século XX.

Entre as facilidades ou dificuldades em trabalhar com PcD (pergunta 05) se observa entre aspectos de dificuldade o índice de $48 \%$, como segundo maior índice; não ter dificuldades em se trabalhar com PcD com $38 \%$; e por último, facilidade o índice foi de $30 \%$. A dificuldade de estrutura do ambiente aparece nas respostas dos participantes com mais frequência, demonstrando que não há ênfase dos processos de gestão, nesses aspectos de inclusão, e dessa forma sendo necessário aplicação de ações como adequação de acessibilidade.

Nos sentimentos com relação à interação com a $\mathrm{PcD}$ (pergunta 06), ainda que apareça como predominante, as pessoas se sentirem bem ou normais nesta interação, ainda aparecem que alguns de sentem responsáveis pela $\mathrm{PcD}$, ou mal e preocupados com essa interação. Desta forma, levantase a necessidade de uma atuação estratégica da área de RH com processos de sensibilização.

$\mathrm{Na}$ pergunta 07, quanto aos comportamentos relevantes nesta interação se observa que não apareceram comportamentos negativos. Respeito e acolhimento, inclusão, referem-se a comportamentos comuns a um bom convívio social. Entre as respostas apareceram que as $\mathrm{PcD}$ precisam se sentir parte integrante do meio, e para isso precisam ser tratadas iguais, apesar de suas diferenças estruturais - física, visual, auditiva ou intelectual. Processo que é compartilhado no conceito de inclusão.

\section{Conclusão}

Esta pesquisa alcançou seu objetivo que era identificar as concepções sobre pessoas com deficiência dos profissionais sem deficiência nas organizações e analisar se interferem nas relações de trabalho. Ficou evidente, nos dados levantados, que a percepção das pessoas sem deficiência, que prevalece sobre as pessoas com deficiência é a de que deveriam se manter segregadas, porém também foram relatadas a necessidade de adaptações e de sensibilização para mais bem recebê-las nas empresas, no que diz respeito à acessibilidade física. Foram abordados com entusiasmo suas contribuições e capacidades de desempenho. E quanto ao relacionamento, esse ocorre de maneira natural e positiva. Pode-se concluir que a inclusão da PcD ocorrerá com êxito nas organizações, desde que o preparo do ambiente e das pessoas seja efetivo.

Acredita-se que o presente trabalho ofereça perspectivas para outros estudos na área. Reconhece-se que ainda há muito a ser pesquisado sobre o assunto, mas a temática das relações entre PcD e suas concepções pode gerar muitos dilemas relativos à gestão de pessoas nas organizações.

\section{Referências}

ARANHA, M.S.F. Trabalho e emprego: instrumento da construção da identidade Pessoal e Social. Brasília: CORDE, 2003.

BARBOSA, L.; DINIZ, D.; SANTOS, W. Diversidade Corporal e Perícia Médica: Novos Contornos da Deficiência para o Benefício da Prestação Continuada. Rev. Textos Contextos, v.8, n.2, p.377390, 2009.

BRASIL, Decreto No 3.298, de 20 de Dezembro de 1999. Regulamenta a Lei $n^{\circ} 7.853$, de 24 de outubro de 1989, dispõe sobre a Política Nacional para a Integração da Pessoa Portadora de Deficiência, consolida as normas de proteção, e dá outras providências. Diário Oficial [da República Federativa do Brasil], Brasília, DF, 1999.

BRASIL, Decreto No 5.296 de 02 de Dezembro de 2004. Regulamenta as Leis nos 10.048 , de 8 de novembro de 2000, que dá prioridade de atendimento às pessoas que especifica, e 10.098, de 19 de dezembro de 2000, que estabelece normas gerais e critérios básicos para a promoção da acessibilidade das pessoas portadoras de deficiência ou com mobilidade reduzida, e dá outras providências. Diário Oficial [da República Federativa do Brasil], Brasília, DF, 02 dez. 2004.

BRASIL. Ministério do Desenvolvimento Social e Combate à Fome. Secretaria Nacional de Assistência Social. LOAS Lei Orgânica de Assistência Social.

CARVALHO-FREITAS, M. N. Análise da Inserção e Gestão do Trabalho de Pessoas com Deficiência: um Estudo de Caso. In: ENANPAD, 2007, Rio de Janeiro. EnANPAD, 2007.

CARVALHO-FREITAS, M. N.; MARQUES, A.L. Concepções de deficiência: as formas de ver a deficiência e suas conseqüências no trabalho. In: CARVALHO-FREITAS, M. N.; MARQUES, A.L. O Trabalho e as pessoas com deficiência: pesquisas, práticas e instrumentos de diagnósticos. Curitiba. Editora Juruá, 2010.

CARVALHO-FREITAS, M.N.; MARQUES, A.L. Instrumentos de Diagnóstico. In: CARVALHO-FREITAS, M.N.; MARQUES, A.L. O trabalho e as pessoas com deficiência: pesquisas, práticas e instrumentos de diagnósticos. Curitiba: Juruá, 2010.

FÁVERO, E.A.G. Direito das pessoas com deficiência: garantia de igualdade na diversidade. Rio de janeiro: WVA, 2004.

GIL, A.C. Gestão de pessoas: enfoque nos papéis profissionais. São Paulo: Atlas, 2009.

IBGE - Instituto Brasileiro de Geografia e Estatística. Censo Demográfico 2010. Disponível em: ftp://ftp.ibge.gov.br/Censos/ Censo_Demografico_2010/Resultados_Gerais_da_Amostra/ tab1.pdf. Acesso em: $\overline{20}$ maio 2018.

MIRANDA, E.C. Papel do líder na busca de resultados por meio do desenvolvimento de pessoas: estudo de caso em área produtiva em uma multinacional do Vale do Paraíba. Taubaté: Universidade de Taubaté, 2005.

UNESCO - Organização das Nações Unidas para a Educação, a Ciência e a Cultura. Declaração de Salamanca e Enquadramento da acção na área das necessidades educativas especiais: acesso 
e qualidade. Salamanca: Ministério da Educação e Ciência de Espanha, 1994.

PEREIRA, A.C.C. Inclusão de pessoas com deficiência no trabalho e o movimento da cultura organizacional: análise multifacetada de uma organização. Porto Alegre: Universidade Federal do Rio Grande do Sul, 2011.

REBELO, P. A pessoa com deficiência e o trabalho. Rio de Janeiro: Qualitymark, 2008.
SASSAKI, R.K. Mídia e deficiência. Brasília: Andi/Fundação Banco do Brasil, 2003,

SASSAKI, R.K. Inclusão. Construindo uma sociedade para todos. Rio de Janeiro: WVA, 1997.

SCHWARZ, A.; HABER J. Cotas: como vencer os desafios da contratação de pessoas com deficiência. São Paulo: i.Social, 2009.

SPECTOR, P.E. Psicologia nas organizações. São Paulo: Saraiva, 2010. 